\title{
PREORDERINGS COMPATIBLE WITH PROBABILITY MEASURES ${ }^{1}$
}

BY

\author{
ROLANDO CHUAQUI AND JEROME MALITZ
}

\begin{abstract}
The main theorem proved in this paper is:
Let $B$ be a $\sigma$-complete Boolean algebra and $\geqslant a$ binary relation with field $B$ such that:

(i) Every finite subalgebra $B^{\prime}$ admits a probability measure $\mu^{\prime}$ such that for $p, q \in B^{\prime}, p \geqslant q$ iff $\mu^{\prime} p \geqslant \mu^{\prime} q$.

(ii) If for every $i, p_{i}, q \in B$ and $p_{i} \subseteq p_{i+1} \preccurlyeq q$, then $\cup_{i<\infty} p_{i} \preccurlyeq q$.

Under these conditions there is a $\sigma$-additive probability measure $\mu$ on $B$ such that:

(a) If there is a $p \in B$, such that for every $q \subseteq p$ there is a $q^{\prime} \subseteq q$ with $q^{\prime} \preccurlyeq q$, $q^{\prime} \nless 0$, and $q \neq q^{\prime}$, then we have that for every $p, q \in B, \mu p \geqslant \mu q$ iff $p \geqslant q$.

(b) If for every $p \in B$, there is a $q \subseteq p$ such that $q^{\prime} \subseteq q$ implies $q \leqslant q^{\prime}$ or $q^{\prime} \leqslant 0$, then we have that for every $p, q \in B, p \geqslant q$ implies $\mu p \geqslant \mu q$.
\end{abstract}

1. The main purpose of this work is to find necessary and sufficient conditions for the existence of $\sigma$-additive probability measures compatible with preorderings (i.e. weak orderings).

These types of considerations are motivated by philosophical, heuristic, and mathematical problems on the foundations of probability. For instance, let $\mathscr{B}$ be the Boolean algebra of events. It is often not feasible, or even possible, to directly assign probabilities to the events. However it may be possible to qualitatively compare events with respect to probability. Thus, we may be able to introduce a preordering $\geqslant$ on $\mathscr{B}$ which can be intuitively interpreted as meaning "qualitatively at least as possible as". In this form the natural question arises about conditions on $\mathscr{B}$ and $\geqslant$ for the existence of a probability measure $\mu$ on $\Re$ such that for all $p, q \in \Re$, we have

$$
p \geqslant q \text { iff } \mu p \geqslant \mu q .
$$

(In this case we say that $\mu$ is compatible with $\succcurlyeq$.) The least we could ask is that

$$
p \geqslant q \text { imply } \mu p \geqslant \mu q .
$$

(For this case we say that $\mu$ is weakly compatible with $\succcurlyeq$.)

There is an extensive literature about the existence of compatible or weakly compatible measures, finitely or $\sigma$-additive. In this paper, we shall concentrate on the $\sigma$-additive case.

A survey of results, especially for the finitely additive case, appears in [KLST, Chapter 5]. We use the following definition of that book.

Received by the editors December 14, 1981 and, in revised form, December 10, 1982.

1980 Mathematics Subject Classification. Primary 60A99; Secondary 03H10, 40J05.

1 This work was supported in part by NSF Grant No. MCS 79-03167 and Organization of American States Grant No. S 63479. 
$\langle\mathscr{B}, \geqslant\rangle$ is a structure of qualitative probability if $\mathscr{B}$ is a Boolean algebra and $\geqslant$ is a binary relation with field $\Re$ such that:

$1 . \geqslant$ is a preorder on $\mathscr{B}$, i.e. it is transitive and connected (complete).

2. $0 * 1$ and $p \geqslant 0$ for every $p \in \Re$.

3. If $p, q, r, \in \mathscr{B}$ and $p \cap q=p \cap r=0$, then $q \geqslant r$ iff $p \cup q \geqslant p \cup r$.

$\langle\Re, \geqslant\rangle$ is a $\sigma$-structure of qualitative probability if $\Re$ is a $\sigma$-complete Boolean algebra and the following holds in addition to 1,2 and 3 .

4. If $p_{i}, q \in \mathscr{B}$ and $p_{i} \subseteq p_{i+1} \preccurlyeq q$ for every $i=0,1,2, \ldots$, then $\cup_{i<\infty} p_{i} \preccurlyeq q$.

$3^{\prime}$. $p \geqslant 0$ and $p \leqslant 0$ for every $p \in \Re$ with $p \neq 0$.

The existence of compatible (or weakly compatible) $\sigma$-measures on $\sigma$-structures of qualitative probability can be reduced to those that are strictly positive (see [SC, $\mathrm{p}$. 361] for a proof of this fact). So from now on we consider only strictly positive $\sigma$-structures of qualitative probability $\langle\Re, \geqslant\rangle$.

Conditions 1-4 are obviously necessary for the existence of a compatible probability measure. Villegas $[\mathbf{V}]$ proved that they are also sufficient in case $\mathscr{B}$ is atomless.

In [SC] it was claimed that they proved this without the assumption that 93 is atomless. However, this theorem is false, as can be easily seen from the example of Kraft, Pratt and Seidenberg [KPS]. This is an example of a finite structure of qualitative probability with no compatible measure (see [KLST, p. 205]).

This example shows that 1-4 are not sufficient in general for the existence of compatible measures. So we add

5. Every finite subalgebra has a compatible measure.

This last condition can be replaced by a purely algebraic condition also due to Kraft, Pratt and Seidenberg [KPS].

$5^{\prime}$. For every $n$ and every $p_{0}, p_{1}, \ldots, p_{n-1} \in \mathscr{B}$ with $\cup_{i<n} p_{i}=1$ and $p_{i} \cap p_{j}=0$ for $i \neq j$, if $q_{0}, \ldots, q_{m-1}$ and $r_{0}, \ldots, r_{m-1}$ are in the subalgebra generated by $p_{0}, \ldots, p_{n-1}$, every $p_{i}$ is contained in the same number of $q_{j}$ 's as $r_{j}$ 's, and $q_{j} \geqslant r_{j}$ for $j=1, \ldots, m-1$, then $r_{0} \preccurlyeq q_{0}$.

The purely atomistic infinite case has a mathematical interest in its own right. Since it can be proved that there are countably many atoms, $\mathscr{B}$ can be viewed as the power set of $\omega, \mathbf{P}(\omega)$. Thus, the problem is to find necessary and sufficient conditions on $\preccurlyeq$ for the existence of a compatible strictly positive $\sigma$-additive probability measure.

We are only able to prove from 1-5 the existence of a weakly compatible strictly positive measure for this case (Theorem 5.3). It remains open whether these conditions are enough for a compatible measure.

On the other hand, when there is a nonempty atomless part, we can prove that 1-5 imply the existence of a compatible measure (Theorem 5.7). Other results of this nature also appear in $\$ \S 5$ and 6.

$\$ 2$ is devoted to preliminaries and contains definitions and elementary facts that will be used throughout the paper.

$\S 3$ is devoted to lemmas, including the fundamental Lemma 3.7, which will be used in proving all the main theorems. 
In $\$ 4$ we consider systems of linear equations and inequalities in nonstandard models of analysis, which, with $\S 3$, provide model theoretic proofs of the theorems in $\S 5$. At this moment we do not know of any purely algebraic-analytic proof.

Finally, in $\$ 7$ we present some open problems and discuss the reasons why we consider them important.

2. Preliminaries. Throughout we let $i, j, k, l, m$, and $n$ denote nonnegative integers. $\mathscr{B}$ is a $\sigma$-Boolean algebra with partial ordering $\subseteq$. $B$ will be identified with its universe and $0,1, \cup, \cup, \cap, \cap, \sim$ have their usual meanings. $p$ and $q$ will denote arbitrary elements of $\mathscr{B}$. We write $p+q$ for $p \cup q$ when $p \cap q=0 ; \Sigma p_{i}$ for $\cup p_{i}$ when $p_{i} \cap p_{j}=0$ for $i \neq j$.

A preordering on $\mathscr{B}$ is a transitive, connected binary relation with field $\mathscr{B}$, i.e., if $p, q, r \in \mathscr{B}$ then either $p \preccurlyeq q$ or $q \preccurlyeq p$, and if $p \preccurlyeq q \preccurlyeq r$ then $p \preccurlyeq r$. We write $p \prec q$ if $p \preccurlyeq q$ but $q \leqslant p ; p \approx q$ if $p \preccurlyeq q$ and $q \preccurlyeq p$.

We introduce binary relation symbols $\prec$ and $\approx$ (confusing these with their intended interpretations in $\mathscr{B}$ ), regard the elements of $\mathscr{B}$ as constant symbols, and let $\Gamma$ be the set of all atomic sentences and negations of atomic sentences in these symbols that are true in $\mathscr{B}$.

A probability measure on $B$ is a $\sigma$-additive nonnegative real valued measure on $\mathscr{B}$ such that $0<\mu(1)<\infty$. $\mu$ is compatible with $\preccurlyeq$ if for all $p, q \in \Re$,

$$
p \preccurlyeq q \text { iff } \mu p \leqslant \mu q .
$$

$\mu$ is weakly compatible with $\preccurlyeq$ if for all $p, q \in \mathscr{B}$,

$$
p \preccurlyeq q \text { implies } \mu p \leqslant \mu q
$$

(allowing the possibility that $p \prec q$ but $\mu p=\mu q$ ).

Throughout the discussion we assume that $\preccurlyeq$ has the following properties.

PROPERTY I. If ' $(B$ ' is a finitely generated subalgebra of $B$ then there is a probability measure $\mu^{\prime}$ on ' $\left(B^{\prime}\right.$ ' that is compatible with $\preccurlyeq$.

PROPERTY II. If $p_{0} \subseteq p_{1} \subseteq \cdots$ and if $p_{1} \preccurlyeq q$ for all $i$, then $\cup p_{i} \preccurlyeq q$.

By taking complements it is easy to see that the following is equivalent to II, so we also denote it as

PROPERTY II. If $p_{0} \supseteq p_{1} \supseteq p_{2} \supseteq \cdots$ and if $p_{i} \geqslant p$ for all $i$, then $\cap p_{i} \geqslant p$.

Note that Properties I and II are necessary for the existence of a probability measure compatible with $\preccurlyeq$.

Property $\mathrm{I}$ is model theoretic in character but can be replaced by an equivalent set of algebraic conditions as described in $\$ 1$.

For reasons mentioned in $\S 1$, we restrict our attention to atomic $\mathscr{B}$ except in Theorems 5.7 and 5.8.

The problem of finding a compatible $\mu$ for the atomic case reduces to that for $\mathscr{B}$ atomic and each atom $a>0$. To see this let $\mathscr{G}$ be the ideal consisting of those $p \approx 0$. Let $\mathscr{B}^{\#}=\mathscr{B} / \mathscr{G}$, the quotient algebra $\mathscr{B} \bmod \mathscr{q}$. If $\mu$ is compatible with $\preccurlyeq / \mathscr{G}$ we obtain a measure $\mu$ compatible with $\preccurlyeq$ on $\mathscr{B}$ by defining $\mu p=\mu^{\#}(p / \mathcal{G})$. So from now on we restrict our attention to atomic $\sigma$-Boolean algebras $\mathscr{B}$ in which $a>0$ for 
each atom. Moreover, since Property I gives a compatible measure for $\mathscr{B}$ finite, we assume that $\mathscr{B}$ has infinitely many atoms.

We next observe that the atoms of $\mathscr{B}$ are arranged in a descending sequence. $a_{0} \geqslant a_{1} \geqslant a_{2} \geqslant \cdots$ with $a_{0}>a_{n+1}$ infinitely often. For suppose that we have atoms $a_{0}, a_{1}, a_{2}, \ldots$ such that $a_{0} \preccurlyeq a_{1} \preccurlyeq a_{2} \preccurlyeq \ldots$. Let $A_{n}=\sum_{n}^{\infty} a_{i}$ for $n=1,2, \ldots$. Clearly $A_{n} \supseteq A_{n+1}$ for all $n$. Moreover, $\cap A_{n}=0$. Hence by II $a_{0} \preccurlyeq 0$, contradicting $a \succ 0$ for all atoms $a$.

We let $S_{n}=\sum_{i>n} a_{i}$.

Unless we state otherwise, we assume all equivalences $p \approx q$ and inequalities $p \succcurlyeq q, p \succ q$ when written are such that $p \subseteq \tilde{q}$.

3. Here we prove several lemmas that will be of use throughout the remainder of the paper. In particular, 3.7 is of central importance.

LEMmA 3.1. Suppose $p>q$. Then there is some $n$ such that $p \cap \tilde{S}_{n} \geqslant q \cap S_{n}$.

Proof. Suppose $p \preccurlyeq q \cup S_{k}$ for all $k$. By Property II, $p \preccurlyeq \cap_{k}\left(q \cup S_{k}\right)$. But $\cap_{k}\left(q \cup S_{k}\right)=q$ so $p \leqslant q$-contradiction. Hence for some $m, p>q \cup S_{m}$. Now suppose $p \cap \tilde{S}_{k} \preccurlyeq q \cup S_{m}$ for all $k$. Again by Property II, $p=\cup_{k}\left(p \cap \tilde{S}_{k}\right) \preccurlyeq q \cup$ $S_{m}$-contradiction. Hence for some $l, p \cap \tilde{S}_{l} \succ q \cup S_{m}$. Now take $n=\min \{l, m\}$.

LeMma 3.2.1. Suppose $p_{0} \prec p_{1} \prec p_{2} \prec \cdots$. Then there is a $q$ such that:

(a) for all $k$ there are infinitely many $l$ such that $q \cap \tilde{S}_{k}=p_{l} \cap \tilde{S}_{k}$; and

(b) $p_{l} \prec q$ for all $l$ and if $p_{l} \prec s$ for all $l$ then $q \preccurlyeq s$.

LEMMA 3.2.2. Suppose $p_{0}>p_{1}>p_{2}>\cdots>s>0$. Then there is a quch that:

(a) for all $k$ there are infinitely many $l$ such that $q \cap \tilde{S}_{k}=p_{l} \cap \tilde{S}_{k}$; and

(b) $p_{l}>q$ for all $l$ and if $p_{l}>r$ for all $l$ then $r \preccurlyeq q$.

Proof OF 3.2.1. We first claim that there is some $a_{n} \subseteq p_{l}$ for infinitely many $l$; for if not, then for each $n$ there is a $k$ such that $p_{l} \subseteq S_{n}$ for all $l \geqslant k$. It follows that for each $l$ and for all $n, p_{l} \preccurlyeq S_{n}$. Hence by Property II each $p_{l} \preccurlyeq \cap S_{n}=0$ and so $p_{l} \approx 0$, contradicting $p_{0} \prec p_{1} \prec p_{2} \prec \cdots$. Hence there is a least $n_{0}$ such that $a_{n_{0}} \subseteq p_{l}$ for infinitely many $l$, say $l_{0}^{0}, l_{1}^{0}, l_{2}^{0}, \ldots$ Let $p_{i}^{1}=p_{l_{i}^{0}}$.

We next claim that there is some $m$ such that $a_{n_{0}}+a_{m} \subseteq p_{i}^{1}$ for infinitely many $i$. If not, then for each $m$ there is a $k$ such that $p_{l}^{l} \subseteq a_{n_{0}} \cup S_{n}$ for all $l \geqslant k$ and, hence, as above, $p_{l}^{1} \preccurlyeq \bigcap_{n}\left(a_{n_{0}} \cup S_{n}\right)$ for each $p_{l}^{1}$ and so $p_{l}^{1} \preccurlyeq a_{n_{0}}$. But $p_{l}^{1} \supseteq a_{n_{0}}$ and so $p_{l}^{1} \approx a_{n_{0}}$ for all $l$, contradicting $p_{0}^{1} \prec p_{1}^{1} \prec \cdots$. Hence there is a least $m$, say $n_{1}$, such that $a_{n_{0}}+a_{n_{1}} \subseteq p_{l}^{1}$ for infinitely many $l$; say $l_{0}^{1}, l_{1}^{1}, l_{2}^{1}, \ldots$

Let $p_{i}^{2}=p_{l_{i}}$ and continue.

Take $q=a_{n_{0}}+a_{n_{1}}+\cdots$.

Clearly $q$ has property (a).

If $q \prec p_{m}$ for some $m$ then $q \cup S_{n} \prec p_{m}$ for some $n$ by 3.1. But then there is a $k$ such that $p_{m} \prec p_{k} \subseteq q \cup S_{n} \prec p_{m}$-contradiction. Hence $p_{m} \preccurlyeq q$ for all $m$.

Suppose $p_{m} \preccurlyeq s$ for all $m$. Then $p_{m} \cap S_{n} \prec s$ for all $m$ and $n$. But for every $n$ there are infinitely many $m$ such that $p_{m} \cap \tilde{S}_{n}=q \cap \tilde{S}_{n}$. Hence $q \cap \tilde{S}_{n} \preccurlyeq s$ for all $n$. By Property II, $q \preccurlyeq s$. This proves (b). 
Proof of 3.2.2. The proof of (a) is similar to that just given for 1 and is left to the reader. To prove (b) suppose that $q>p_{n}$ for some $n$. By Lemma 3.1 there is a $k$ such that $q \cap \tilde{S}_{k}>p_{n}$ and so $q \cap \tilde{S}_{k}>p_{l}$ for all $l \geqslant k$. But by (a) there is an $l$ such that $q \cap \tilde{S}_{k} \subseteq p_{l}$ and $l \geqslant k$, and so $q \cap \tilde{S}_{k} \preccurlyeq p_{l}$-contradiction. Hence $q \preccurlyeq p_{n}$ for all $n$.

Suppose $r \preccurlyeq p_{n}$ for all $n$. It follows from (a) that $r \preccurlyeq q \cap \tilde{S}_{n}$ for all $n$. Hence $r \preccurlyeq q$, as needed to complete (b).

LEMMA 3.3. Suppose $p+p_{0}>q+q_{0}$ where $p \cup q \subseteq \tilde{S}_{n}$ for some $n, p_{0}, q_{0} \subseteq S_{n}$ and $p+p_{1} \approx q+q_{1}$ for all $p_{1}, q_{1} \subseteq S_{n}$. Then there is some $p^{1} \preccurlyeq p_{0}$ and some $q^{1} \succcurlyeq q_{0}, p^{1}$, $q^{1} \subseteq S_{n}$, such that:

(i) $p+p_{0} \geqslant p+p^{1}>q+q^{1} \geqslant q+q_{0}$; and

(ii) if $p+p_{0} \geqslant p+p^{*} \succ q+q^{*} \geqslant q+q_{0}$, with $p^{*}, q^{*} \subseteq S_{n}$, then $p^{*} \geqslant p^{1}$ and $q^{*} \preccurlyeq q^{1}$.

Proof. Let $K=\left\{q^{*}: p+p_{0}>q+q^{*}\right.$ and $S_{n} \supseteq q^{*} \supseteq S_{m}$ for some $\left.m\right\}$. Notice that $K$ is countable and, if $p+p>q+q^{*}$, with $q^{\#} \subseteq S_{n}$, then $q^{\#} \subseteq q^{*}$ for some $q^{*} \in K$ by 3.1 .

We claim that there is a $q^{1} \in K$ such that $q^{1} \geqslant q^{*}$ for all $q^{*} \in K$. If not then there is an infinite sequence $q_{1} \prec q_{2} \prec q_{3} \prec \cdots$ cofinal in $K$. By Lemma 3.2 there is a $q^{\#}$ such that $p+p>q+q^{\#}$ and each $q_{i} \prec q^{\#}$. Then by Lemma 3.1 there is a $k$ such that $p+p_{0}>q+\left(q^{\#} \cup S_{k}\right)$. We let $q^{1}=q^{\#} \cup S_{k}$. Clearly $q^{1}$ is the maximal member of $K$.

Now let $L=\left\{p^{*}: p+p^{*}>q+q^{1}, p^{*} \subseteq S_{n}\right.$ and $p^{*}$ finite $\}$. Clearly $L$ is countable and by Lemma 3.1 if $p+p^{\#}>q+q^{1}$, with $p^{\#} \subseteq S_{n}$, then there is some $p^{*} \in L$ such that $p^{*} \subseteq p^{\#}$.

We claim that $L$ has a minimal member. For if not, then there is an infinite decreasing sequence $p_{1}>p_{2}>p_{3}>\cdots$ cofinal in $L$. If there is no $r$ such that $p_{i}>r$ for all $i$ then clearly for each $n$ there is a $k$ such that for all $l \geqslant k, p_{l} \subseteq S_{m}$. So for $l$ large enough, $p_{l} \subseteq S_{m}$ and $p+S_{m}>q+q^{1}$. Hence by Property II we have $p>q+$ $q_{1}$ and so $p^{1}$ can be taken as 0 , which is in $L$. If there is some $r$ for which $0 \prec r \prec p_{i}$ for each $i$, take $p^{\#}$ to be the $q$ of Lemma 3.2. Since, for each $m, p^{\#} \cup S_{m} \supseteq p_{i}$ for some $i$ we have $p+\left(p^{\#} \cup S_{m}\right)>q+q^{1}$. Hence, by Property II, $p+p^{\#}>q+q^{1}$. By 3.1, $p+p^{1} \succ q+q^{1}$ for some finite $p^{1} \subseteq p^{\#}$. But then $p^{1} \in L$ and is minimal with respect to ' $\prec$,

It is now easy to see that (i) and (ii) are satisfied.

LEMMA 3.4. Suppose $p \prec q, p, q \subseteq \tilde{S}_{n}$ and for no $p^{\#}, q^{\#} \subseteq S_{n}$ do we have $p+p^{\#} \approx$ $q+q^{\#}$. Then it is possible to find finitely many pairs $\left\{\left\langle p^{i}, q^{i}\right\rangle\right.$ for $\left.i \leqslant n\right\}$, with $p^{i}, q^{i} \subseteq S_{n}$, such that:

(i) $p+p^{i}>q+q^{i}$ for each $i$; and

(ii) whenever $p+p_{0} \succ q+q_{0}, p_{0}, q_{0} \subseteq S_{n}$, then there is an $i \leqslant n$ such that $p_{0} \geqslant p^{i}$ and $q_{0} \preccurlyeq q^{i}$.

Proof. Let $K=\left\{\left\langle p^{\prime}, q^{\prime}\right\rangle: p+p^{\prime}\right\rangle q+q^{\prime}$, and if $p^{\prime \prime} \leqslant p^{\prime}$ and $q^{\prime \prime} \geqslant q$ and $p+p^{\prime \prime}>q+q^{\prime \prime}$, then $p^{\prime \prime} \approx p^{\prime}$ and $\left.q^{\prime \prime} \approx q^{\prime}\right\}$. Note that Lemma 3.3 shows that for 
any $p^{\prime \prime}$ and $q^{\prime \prime}$ such that $p+p^{\prime \prime}>q+q^{\prime \prime}$, there is a $\left\langle p^{\prime}, q^{\prime}\right\rangle \in K$ such that $p^{\prime \prime} \geqslant p^{\prime}$ and $q^{\prime \prime} \preccurlyeq q^{\prime}$.

For $\left\langle p_{0}, q_{0}\right\rangle,\left\langle p_{1}, q_{1}\right\rangle \in K$ define $\left\langle p_{0}, q_{0}\right\rangle \sim\left\langle p_{1}, q_{1}\right\rangle$ if $p_{0} \approx p_{1}$ and $q_{0} \approx q_{1}$. This defines an equivalence relation on $K$ and we let $K^{\prime}$ consist of one representative from each equivalence class. Suppose $\left\langle p_{0}, q_{0}\right\rangle$ and $\left\langle p_{1}, q_{1}\right\rangle$ are distinct members of $K^{\prime}$. We can $n o t$ have either

(i) $p+p_{0} \geqslant p+p_{1}>q+q_{1} \geqslant q+q_{0}$ or

(ii) $p+p_{0} \geqslant p+p_{1} \geqslant q+q_{0} \geqslant q+q_{1}$ since both imply $\left\langle p_{0}, q_{0}\right\rangle \sim\left\langle p_{1}, q_{1}\right\rangle$. Hence we must have either

$$
p+p_{0}>q+q_{0} \geqslant p+p_{1}>q+q_{1}
$$

or

$$
p+p_{1}>q+q_{1} \geqslant p+p_{0}>q+q_{0} \text {. }
$$

If the first holds we say that $\left.\left\langle p_{0}, q_{0}\right\rangle\right\rangle\left\langle p_{1}, q_{1}\right\rangle$. So $>$ linearly orders $K^{\prime}$. Also, by 3.1 and the definition of $K$ we see that $K^{\prime}$ is countable.

It will be enough to show that $K^{\prime}$ is finite.

Claim (1). There is no infinite increasing chain in $K^{\prime}$ of the form

$$
\cdots \geqslant p+p^{1}>q+q^{1} \geqslant p+p^{0}>q+q^{0} .
$$

Otherwise, by Lemma 3.2, there is a $p^{\#}$ and a $q^{\#}$ such that for all $n, p^{\#} \cap \tilde{S}_{n} \subseteq p^{i}$ for infinitely many $i$ and $q^{\#} \cap \tilde{S}_{n} \subseteq q^{i}$ for infinitely many $n$. Clearly, $p+\left(p^{\#} \cup\right.$ $\left.S_{m}\right)>q+\left(q^{\#} \cap \tilde{S}_{n}\right)$ for all $m$ and $n$. Two applications of Property II give $p+p^{\#}$ $\succcurlyeq q+q^{\#}$ and, since $p+p^{\#} \approx q+q^{\#}$ is ruled out by hypotheses, $p+p^{\#}>q+q^{\#}$. Hence, for some $m$ and $n, p+\left(p^{\#} \cap \tilde{S}_{m}\right)>p+\left(q^{\#} \cup S_{m}\right)$. But $q^{\#} \cup S_{m}>q^{i}$ for all $i$, and for some $\left\langle p^{*}, q^{*}\right\rangle \in K$ we have $q^{*} \geqslant q^{\#}$-contradiction. This establishes Claim (1).

Claim (2). There is no infinite decreasing cofinal chain in $K^{\prime}$ of the form

$$
p+p^{0}>q+q^{0} \geqslant p+p^{1}>q+q^{1} \geqslant p+p^{2}>q+q^{2} \geqslant \cdots .
$$

If $q^{i}>r$ for some $r>0$ and all $i$, let $q^{\#}$ be the $q$ of Lemma 3.2. Otherwise let $q^{\#}=0$. Similarly, applying 3.2 to $p^{0}>p^{1}>\cdots$ we get $p^{\#}$. Since for all $n$ there is a $k$ such that $p+\left(p^{\#} \cup S_{n}\right)>q+q^{k}>q+q^{\#}$, it follows by Property II that $p+p^{\#}>q+q^{\#}$. Take $n$ such that $p+\left(p^{\#} \cap \tilde{S}_{n}\right)>q+\left(q^{\#} \cup S_{n}\right)$ by 3.1. There is an $m$ such that $p^{m} \supseteq p^{\#} \cap \tilde{S}_{n}$ and $q^{m} \prec q^{\#} \cup S_{n}$. But this is impossible since whenever $p^{m} \geqslant p^{\prime \prime}$ and $q^{m} \preccurlyeq q^{\prime \prime}$ and $p+p^{\prime \prime}>q+q^{\prime \prime}$, then $p^{m} \approx p^{\prime \prime}$ and $q^{m} \approx q^{\prime \prime}$. Hence $K^{\prime}$ has no infinite descending sequence, giving Claim 2.

The two claims now establish the finiteness of $K^{\prime}$, completing the proof.

The next lemma has the same form as the preceding except the initial condition $p \prec q$ is replaced by $p \succ q$.

Lemma 3.5. Suppose $p>q, p, q \subseteq \tilde{S}_{n}$ and for no $p^{\#}, q^{\#} \subseteq S_{n}$ do we have $p+p^{\#} \approx$ $q+q^{\#}$. Then it is possible to find finitely many pairs $\left\{\left\langle p^{i}, q^{i}\right\rangle: i \leqslant n\right\}$ such that $p^{i}, q^{i} \subseteq S_{n}$,

(i) $p+p^{i}>q+q^{i}$ for each $i \leqslant n$, and

(ii) if $p+p_{0}>q+q_{0}$, with $p_{0}, q_{0} \subseteq S_{n}$, then for some $i \leqslant n$ we have $p_{0} \geqslant p^{i}$ and $q_{0} \preccurlyeq q^{i}$ 
Proof. Let $K$ and $K^{\prime}$ be as in Lemma 3.4. As before we see that the members of $K^{\prime}$ form a countable linear ordering given by $\left\langle p^{i}, q^{i}\right\rangle>\left\langle p^{j}, q^{j}\right\rangle$ iff $\left.p+p^{i}\right\rangle q+$ $q^{i} \geqslant p+p^{j}>q+q^{j}$, and the ordering has no infinite increasing subsequence in $K^{\prime}$. The proof will be completed by showing that there is no infinite decreasing subsequence in $K^{\prime}$.

Using Lemma 3.2 get $q^{*}$ such that $p>q+q^{*}$, and whenever $p>q+q^{\#}$, then $q^{*} \geqslant q^{\#}$. Clearly, $\left\langle 0, q^{*}\right\rangle \in K$ and we can choose $K^{\prime}$ so as to have $\left\langle 0, q^{*}\right\rangle \in K^{\prime}$. We cannot have $\left\langle p_{0}, q_{0}\right\rangle \in K^{\prime}$ with $\left\langle 0, q^{*}\right\rangle>\left\langle p_{0}, q_{0}\right\rangle$, for otherwise $\left.p\right\rangle p+p_{0}$. Hence, if there is an infinite decreasing chain it must look like

$$
p+p^{0}>q+q^{0} \geqslant p+p^{1}>q+q^{1} \geqslant p+p^{2}>q+q^{2} \geqslant \cdots \geqslant p>q+q^{*} .
$$

By Lemma 3.2 we get $q^{\#}$ such that for any $n$ there are infinitely many $i$ such that $q^{\#} \cap \tilde{S}_{n}=q^{i} \cap \tilde{S}_{n}$. (Note that 3.2 yields such a $q^{\#}$ since $q^{i}>q^{*}$ for all $i$.) Again by 3.1 get $p^{\#}$ such that for each $n$ there are infinitely many $i$ such that $p^{\#} \cap \tilde{S}_{n}=p^{i} \cap$ $\tilde{S}_{n}$. Several applications of 3.1 give a $k$ and an $l$ such that

$$
p+\left(p^{\#} \cap \tilde{S}_{k}\right)>q+\left(q^{\#} \cup S_{l}\right) \text {. }
$$

For some $m, p^{m} \supseteq p^{\#} \cap \tilde{S}_{k}$ and $p^{m} \neq p^{\#} \cap \tilde{S}_{k}$ and $q^{\#} \cup S_{l} \supseteq q^{m}$ and $q^{\#} \cup S_{l} \neq$ $q^{m}$. But then

$$
p+p^{m}>p+\left(p^{\#} \cap \tilde{S}_{k}\right)>q+\left(q^{\#} \cup S_{l}\right)>q+q^{m},
$$

contradicting $\left\langle p^{m}, q^{m}\right\rangle \in K$.

DEFINITION 3.6. Let $I_{n}=\left\{p: p \subseteq a_{0}+a_{1}+\cdots+a_{n}\right\}$ and $T_{n}=\{p: p \subseteq$ $\left.\sum_{i=n+1}^{\infty} a_{i}\right\}$. Let $R$ be the first order theory of the reals with + and $<$. For $\Omega \subseteq \Gamma$ let $\Omega^{\#}=\{p<q: p \prec q \in \Omega\} \cup\{p=q: p \approx q \in \Omega\}$. Let $\Sigma_{n}=R \cup \Sigma_{n}^{-}$, where $\Sigma_{n}^{-}$is the set of all expressions of the form $p<q$ or $p=q$, where $p, q \in T_{n}$, that can be deduced from $R \cup \Gamma^{\#}$.

LEMMA 3.7. For every $n$ there is a finite subset $\Delta_{n} \subseteq \Gamma$ such that $\Delta_{n}^{\#} \cup \Sigma_{n} \vDash \Gamma^{\#}$.

Proof. We first define $\Delta_{n}$. Let $p, q \in I_{n}$.

(1) If $p \prec q$ is in $\Gamma$ put $p \prec q$ in $\Delta_{n}$. If $p \approx q$ is in $\Gamma$ put $p \approx q$ in $\Delta_{n}$.

(2) If for some $p_{0}, q_{0} \in T_{n}$ we have $p+p_{0} \approx q+q_{0}$, put one such instance in $\Delta_{n}$.

(3) If for no $p_{0}, q_{0} \in T_{n}$ do we have $p+p_{0} \approx q+q_{0}$, then put all of the finitely many expressions $p+p^{i}>q+q^{i}$ arising in Lemmas 3.4 and 3.5 into $\Delta_{n}$.

Clearly, any equality $p+p^{1} \approx q+q^{1}$ in $\Gamma$ is derivable from $\Delta_{n} \cup \Sigma_{n}$ by (1) and (2).

Now suppose $p+p_{0} \succ q+q_{0}$ is in $\Gamma$ with $p, q \in I_{n}$ and $p_{0}, q_{0} \in T_{n}$. Three cases arise.

Case (1). There is some $p^{1}, q^{1} \in T_{n}$ such that $p+p^{1} \approx q+q^{1}$. In this case there is a $p^{\prime \prime} \in T_{n}$ such that $p+p^{\prime \prime}=q+q^{\prime \prime}$ is in $\Delta_{n}^{\#}$. In $\Sigma_{n}$ we have $q^{\prime \prime}+p_{0}>p^{\prime \prime}+q_{0}$ (note that the plus signs in the last expression refer to usual addition in $R$ and not to disjoint union in $\mathscr{B}$ ). From this it follows that $\Delta_{n}^{\#} \cup \Sigma_{n} \vDash p+p_{0}>q+q_{0}$.

Case (2). $p \prec q$ and for no $p^{1}, q^{1} \in T_{n}$ do we have $p^{1}+p \approx q^{1}+q$. By Lemma 3.4 there is some $p^{*}$ and $q^{*}$ such that $p+p_{0} \geqslant p+p^{*}>q+q^{*} \geqslant q+q_{0}$, where 
$p+p^{*}>q+q^{*}, p_{0} \geqslant p^{*}, q^{*} \leqslant q_{0}$ are all in $\Delta^{\#} \cup \Sigma_{n}$. From this it follows that $\Delta_{n}^{\#} \cup \Sigma_{n} \vDash p+p_{0}>q+q_{0}$.

Case (3). $p>q$ and for no $p^{1}, q^{1} \in T_{n}$ do we have $p^{1}+p \approx q^{1}+q$. This is handled similarly to (2) by using 3.3 .

4. In this section we consider finite systems of inequalities in nonstandard models of analysis.

LEMMA 4.1. Suppose that $\delta$ is a finite system of equalities of the form

$$
\sum_{j=1}^{m} \alpha_{i j} x_{j}=c_{j}
$$

for $j \leqslant k$, and each $\alpha_{i j}$ is finite and not infinitesimal or zero, and each $c_{j}$ is finite. If $\delta$ has a nonstandard solution it has a finite solution. (Finite in a nonstandard model means not infinite.)

Proof. Easy by a Gaussian elimination of variables.

Lemma 4.2. Suppose that $\delta$ is a finite system of equalities and inequalities, say $\mathcal{S}=\mathfrak{S}_{1} \cup \mathcal{S}_{2}$, where

$$
\S_{1}=\left\{\sum_{j=1}^{m} \alpha_{i j} x_{j}=c_{j}: j=1, \ldots, k\right\}
$$

and

$$
\delta_{2}=\left\{\sum_{j=1}^{m} \beta_{i j} x_{j} \Delta_{j} d_{j}: j=1, \ldots, l\right\}
$$

where $\Delta_{j}$ is $<$ or $\leqslant$. Also suppose that each $\alpha_{i j}$ and each $\beta_{i j}$ is finite and not infinitesimal, and each $c_{j}$ and $d_{j}$ is finite. If $\delta$ has a solution then $\delta$ has a finite solution.

Proof. By induction on $l$. If $l=0$ the lemma reduces to 4.1. Suppose the statement is true for $l=n$ and let $l=n+1$. Let $C$ be the solution set for $\mathcal{S}_{1} \cup \mathcal{S}^{-}$, where $\delta^{-}=\left\{\sum \beta_{i j} x_{i} \Delta_{j} d_{j}: j=1,2, \ldots, n\right\}$. Let $\sigma$ be the remaining inequality $\sum \beta_{n+1, j} x_{j}>d_{j}$ (or $\geqslant d_{j}$ ). Let $H$ be the half-space determined by $\sigma$. Three cases arise.

Case (1). $H \cap C=\varnothing$. This is impossible since $\delta$ has a solution.

Case (2). $H \supseteq C$. Then we are done since our induction hypothesis says that $C$ has finite solutions.

Case (3). There are points $r$ and $s$ such that $r \in H \cap C$ and $s \in \tilde{H} \cap C$.

Since $C$ is convex, the line segment $L$ joining $r$ to $s$ is contained in $C$. Hence there is a point $t$ on $L$ lying on the boundary of $H$. This point satisfies $\delta^{\#}$ where $\mathcal{S}^{\#}=\mathcal{S}_{1} \cup \mathcal{S}^{-} \cup\left\{\sigma^{\#}\right\}$ and $\sigma^{\#}$ is $\Sigma \beta_{n+1, j} x_{j}=d_{j}$.

Since $\delta^{\#}$ has only $n$ inequalities, it has a finite solution $r^{\#}$. Hence, if $\sigma$ is a weak inequality we are done.

If $\sigma$ is a strong inequality we consider the line segment $L^{\#}$ joining $r$ to $r^{\#}$. Note that $L^{\#} \subseteq C \cap \bar{H}$, where $\bar{H}$ is the closure of $H$.

If $r$ is finite then $r$ satisfies $\delta$. 
If $r$ is infinite then $L^{\#}$ is infinite in length and the point $r^{1}$ of distance 1 from $r^{\#}$ along $L^{\#}$ satisfies $\mathcal{S}$. (Note that $r^{1}$ is not on the boundary of $H$, otherwise $L^{\#}$ would be contained in the boundary, contradicting the fact that $r$ is not on the boundary.)

5. In this section we prove the existence of probability measures weakly compatible with $\preccurlyeq$.

Let $\mathscr{R}=\langle\mathbf{R},+, \cdot\rangle$ be the reals with addition and multiplication.

LEMma 5.1. For each $i$ there is a structure $M_{i} \equiv \Re$ and a function $\mu_{i}$ on $\bigcirc$ into $M_{i}$ such that:

(i) $\mu_{i} p \leqslant \mu_{i} q$ iff $p \leqslant q$;

(ii) $\mu_{i} a_{i}$ is not infinitesimal;

(iii) $\mu_{i} a_{0}$ is finite and greater than 0.

Proof. Let $\Sigma=$ Th $\leftrightarrow \cup \Gamma^{\#}$, where $\Gamma^{\#}$ is as in 3.6. By first order compactness, $\Sigma$ has a model $M$.

If $a_{i}^{M}$ is not infinitesimal, take $M_{i}=M$ and for each $p \in \mathscr{B}$ let $\mu_{i} p=p^{M_{i}}$.

Suppose that $a_{i}^{M}$ is infinitesimal.

We first note that there is some standard natural number $n$ such that $\left(n a_{i}\right)^{M}>S_{i}^{M}$. Indeed, it follows from Property II that $a_{i}>S_{j}$ for some $j>i$. Hence $S_{i}=\left(a_{i+1}\right.$ $\left.+\cdots+a_{j}+S_{j}\right) \leqslant(j-i+1) a_{i}$.

Let $\nu p=\left(p / a_{i}\right)^{M}$. Note that $\nu$ satisfies (i) and (ii).

Let $S$ be the system of equalities and inequalities obtained from $\Delta_{i-1}$ by replacing $a_{j}$ by a variable $x_{j}$ for $j \leqslant i-1$ and each $p \in T_{i-1}$ by $\nu p$. $\delta$ has a solution in $M$; indeed assigning $\nu a_{j}$ to $x_{j}$ gives us a solution. Hence, by Lemma 4.2 there is a finite solution, say the assignment of $r_{j}$ to $x_{j}$. Now let $\mu_{i} a_{j}=r_{j}$ for $j<i$ and $\mu_{i} a_{k}=\nu a_{k}$ for $k \geqslant i$. If $p=p_{0}+p_{1}$ with $p_{0} \in I_{i}$ and $p_{1} \in T_{i}$, define $\mu_{i} p=\mu_{i} p_{0}+\nu p_{1}$, where $\mu_{i} p_{0}=\Sigma\left\{\mu_{i} a_{k}: a_{k} \subseteq p_{0}\right\}$. Clearly this assignment satisfies $\Delta_{i-1}^{\#} \cup \Sigma_{i}$ and, hence, by $3.7, \Gamma^{\#}$ is satisfied. It follows that (i), (ii) and (iii) are satisfied.

LEMMA 5.2. For each $i$ there is a standard probability measure $\bar{\mu}_{i}$ on $\Re$ such that

(i) $\bar{\mu}_{i} a_{i}>0$,

(ii) $p \geqslant q$ implies $\bar{\mu}_{i} p \geqslant \bar{\mu}_{i} q$.

Proof. Let $\mu_{i}$ be as in Lemma 5.1. Let $\bar{\mu}_{i} p$ be the standard part of $\mu_{i} p$ for each $p \in \mathscr{B}$. Clearly (i) and (ii) are satisfied; also $\bar{\mu}_{i}$ is finite, positive, and finitely additive. It is enough to show that $\bar{\mu}_{i}$ is monotone. This is clear if $\bar{\mu}_{i} a_{j}=0$ for some $j$, since then $\bar{\mu}_{i} a_{j+k}=0$ for all $k$ and the finite additivity of $\bar{\mu}_{i}$ gives $\sigma$-additivity.

On the other hand, suppose $\bar{\mu}_{i} a_{j}>0$ for all $j$. Consider a sequence $p_{0} \subseteq p_{1} \subseteq p_{2}$ $\subseteq \cdots$ such that $\bar{\mu}_{i} p_{j} \leqslant \bar{\mu}_{i} q$ for all $i$.

Case (1). If $\mu_{i} p_{j} \leqslant \mu_{i} q$ for all $j$ then $p_{j} \preccurlyeq q$ for all $j$, so $\cup_{j} p_{j} \preccurlyeq q$. Hence, $\mu_{i}\left(\cup_{j} p_{j}\right) \preccurlyeq \mu_{i} q$, so $\bar{\mu}_{i}\left(\cup_{j} p_{j}\right) \leqslant \bar{\mu}_{i} q$, as needed.

Case (2). Suppose $\mu_{i} p_{j}>\mu_{i} q$ for some $j$. Then $\mu_{i} p_{j} \leqslant \mu_{i} p_{j+k}$ for all $k$, yet $\bar{\mu}_{i} p_{j}=\bar{\mu}_{i} q$, so $\mu_{i} p_{j}$ and $\mu_{i} p_{j+k}$ differ by an infinitesimal for all $k$. Since $\mu_{i} a_{l}$ is not infinitesimal for all $l$ and since $\mu_{i}$ is finitely additive, $p_{j}=p_{j+k}$ for all $k$. Hence, $p_{j}=\cup_{t} p_{t}$, so $\bar{\mu}_{i} \cup_{t} p_{t}=\bar{\mu}_{i} q$. 
THEOREM 5.3. There is a standard probability measure $\mu$ on $\Re$ such that:

(i) $\mu a_{i}>0$ for all $i$;

(ii) $p \geqslant q$ implies $\mu p \geqslant \mu q$;

(iii) if $p>q$ and if there are infinitely many $n$ such that $a_{n} \not q$, then $\mu p>\mu q$.

Proof. Let $\mu p=\sum_{i=0}^{\infty} \bar{\mu}_{i}(p) \cdot 2^{-(i+1)}$, where $\bar{\mu}_{i}$ is as in 5.2. Clearly $\mu$ satisfies (i) and (ii). Suppose $p>q$ and $a_{n} \nsubseteq q$ for infinitely many $n$. By 3.1 there is some $k$ such that $p>q \cup S_{k}$, and so some $a_{n}$ such that $p>q+a_{n}$. By (ii) $\mu p \geqslant \mu q+\mu a_{n}$. By (i) $\mu p>\mu q$.

Corollary 5.4. Let $b_{0}, b_{1}, \ldots$ be a subsequence of $a_{0}, a_{1}, \ldots$ such that $\sum b_{i} \nsupseteq S_{n}$ for all $n$. Let $\Re^{-}$be the $\sigma$-subalgebra of $\Re$ with elements $\left\{p: p \subseteq \sum b_{i}\right\}$. Then there is $a$ probability measure $\mu$ on $\mathscr{B}^{-}$such that for all $p, q \in \Re^{-}$,

$$
p \geqslant q \text { iff } \mu p \geqslant \mu q .
$$

Proof. This follows immediately from the previous theorem, noting that (iii) will be satisfied by any $q \in \Re^{-}$.

COROLlARY 5.5. There is a (nonstandard) finitely additive measure $\mu$ such that

(i) $p \geqslant q$ iff $\mu p \geqslant \mu q$,

(ii) $\mu a_{i}$ is not infinitesimal for any $i$.

Proof. The following sentences are consistent with first order analysis.

(1) $f(m, x)$ has domain $N \times \mathscr{B}$ and range $\subseteq[0,1]$ (where $N$ is the set of natural numbers).

(2) For each $i \in N, f(i, p) \geqslant f(i, q)$ iff $p \geqslant q$.

(3) $f\left(j, a_{j}\right)>r_{j}$, where $r_{j}$ is some standard rational number such that $\mu_{j} a_{j}>r_{j}>0$ and $\mu_{j}$ is as in Lemma 5.1.

(4) $F(p)=\Sigma_{j \in N_{2}} f(j, p) /(j+2)$.

We consider a model $M$ for these sentences along with a suitable portion of first order analysis and let $\mu p$ be the value of $F(p)$ in $M$. Clearly (i) and (ii) are satisfied.

DEFINITION 5.6. Let $\Im$ be a $\sigma$ Boolean algebra with a countable number of atoms $a_{0}, a_{1}, a_{2}, \ldots$ Let $\mathscr{B}^{\prime}$ be the subalgebra generated by the atoms and let $\mathscr{B}^{\prime \prime}$ be the subalgebra consisting of those $b \in \mathscr{B}$ such that $b \subseteq B^{\prime \prime}$, where $\tilde{\mathscr{B}}^{\prime \prime}=\sum a_{i}$. Let $B^{\#}=\left\{a_{i}: a_{i} \preccurlyeq B^{\prime \prime}\right\}$ and let $\mathscr{B}^{*}$ be the smallest $\sigma$-algebra containing $B^{\#}=\{p$ : $\left.p \subseteq B^{\prime \prime}\right\}$.

THEOREM 5.7. Let $\mathscr{B}$ be such that neither $\mathscr{B}^{\prime}$ nor $\mathfrak{B}^{\prime \prime}$ is empty. Then there is a strictly positive probability measure $\mu$ on $\mathscr{B}$ compatible with $\succcurlyeq$. Moreover, if $\nu$ is another such measure then there is a constant $c>0$ such that for all $p \in \mathscr{B}^{*}, \nu p=c \mu p$.

Proof. By a lemma in [SC] there is a sequence $b_{0}, b_{1}, b_{2}, \ldots$ such that $\mathscr{B}^{\prime \prime}=\sum b_{i}$ and $b_{i} \approx \sum_{j} b_{i+1+j}$ for all $i$. Let $\mathscr{B}^{-}$be the atomic subalgebra generated by $\left\{a_{0}, a_{1}, \ldots\right\} \cup\left\{b_{0}, b_{1}, \ldots\right\}$. Let $\Gamma^{-}$be the set of all expressions $p \geqslant q$ and $p>q$ in $\Gamma$ such that $p, q \in \mathscr{B}^{-}$. By Theorem 5.5 there is a nonstandard measure $\nu^{-}$on $\mathscr{B}$ that is compatible with $\succcurlyeq$.

Let $\nu^{-} p=\left(\nu^{-} B^{\prime \prime}\right)^{-1} \nu p$ for all $p \in \Re^{-}$.

Of course $\mu^{-}\left(B^{\prime \prime}\right)=1, \mu^{-} b_{i}=1 / 2^{i+1}$ and $\mu^{-}$is compatible with $\succcurlyeq$. 
By Property II there is an $S_{m} \preccurlyeq B^{\prime \prime}$, and hence there is some $a_{n} \preccurlyeq B^{\prime \prime}$.

We claim that $\mu^{-} a_{n}$ is standard for all $a_{n} \preccurlyeq B^{\prime \prime}$. To see this it is enough to show that $a_{n} \approx \Sigma_{i \in I} b_{i}$ for some $I$. Let $b^{k}=\sum_{i \geqslant k} b_{i}$. Since $\cap_{k} b^{k}=0$, Property II implies that $a_{n}>b^{k}$ for some $k$ and, hence, $a_{m}>b_{l}^{1}$ for some $l$. Let $b_{0}^{1}$ be the first such. If $a_{n} \approx b_{0}^{1}$, we are done; if $a_{n}>b_{0}^{1}$ there is some $b_{l}$ such that $a_{n} \geqslant b_{0}^{1}+b_{l}$ (another use of Property II). This gives $b_{1}^{1}$ with $a_{n} \geqslant b_{0}^{1}+b_{1}^{1}$. Continue the process to get $b_{0}^{1}, b_{1}^{1}, \ldots$ If the process ends in finitely many steps, we are done. Otherwise we get $a_{n} \geqslant \sum b_{i}^{1}$. In this case we must get $\approx$. For if $a_{n} \approx B^{\prime \prime}$ there is some $b_{i} \not \Sigma b_{i}^{1}$. If there are infinitely many $b_{j} \nsubseteq \Sigma b_{i}^{1}$, then a slight modification of the proof of 3.1 gives $a_{n}>\sum b_{i}^{1}+b_{j}$ for some $j$, which is impossible by the definition of the $b_{i}^{1}$ 's. Otherwise there is a $k$ such that $\sum b_{i}^{1} \approx \sum_{j \in J} b_{j}^{1}+b^{k+1}$, where $b_{k} \notin\left\{b_{j}^{1} \mid J \in J\right\}$. But then $\Sigma b_{i}^{1}=\Sigma_{j \in J} b_{j}^{1}+b_{k}$, again an impossibility by the definition of the $b_{i}^{1}$ 's.

Let $n$ be the largest $m$ such that $a_{m} \preccurlyeq B^{\prime \prime}$. Let $\mathcal{S}$ be the system of equalities and inequalities obtained from $\Delta_{n}$ by replacing $a_{j}$, with $j \leqslant n$, by a variable $x_{j}$, and each $p \in T_{n}$ by $\mu^{-} p$. $\delta$ has a solution in a nonstandard model and so has a solution in the reals, say one that assigns $r_{j}$ to $x_{j}$.

Now define $\mu p=\mu^{-} p$ for all $p \in T_{n}$ and $\mu \Sigma^{k} a_{i_{j}}=\Sigma^{k} r_{i_{j}}$ when $\Sigma^{k} a_{i_{j}} \in I_{n}$. The conditions of Lemma 3.7 are met and so $\mu$ is compatible with $\geqslant$ on $\mathscr{B}^{-}$.

Let $\lambda$ be the unique probability measure on $\mathscr{B}^{\prime \prime}$ that is compatible with $\succcurlyeq$ restricted to $\Re^{\prime \prime}$. Clearly $\lambda b_{i}=\mu b_{i}$ for all $i$. Now extend $\mu$ to all of $\mathscr{B}$ by defining $\mu\left(p_{0}+p_{1}\right)=\mu p_{0}+\lambda p_{1}$ when $p_{0} \in \mathscr{B}^{\prime}$ and $p_{1} \in \mathscr{B}^{\prime \prime}$.

We claim that $\mu$ is compatible with $\geqslant$. For suppose $p=p_{0}+p_{1}, q=q_{0}+q_{1}$, $p>q, p_{0}, q_{0} \in \mathscr{B}^{\prime}$ and $p_{1}, q_{1} \in \mathscr{B}^{\prime \prime}$. Then there is some $p_{1}^{1}$ and $q_{1}^{1}$ such that $p_{1}^{1} \approx p_{1}$, $q_{1}^{1} \approx q_{1}$, and $p_{1}^{1}=\sum_{i \in I} b_{i}, q_{1}^{1}=\sum_{j \in J} b_{j}$ for some $I$ and $J$. But $p_{0}+p_{1}^{1}>q_{0}+q_{1}^{1}$ and $p_{0}+p_{1}^{1}, q_{0}+q_{1}^{1} \in \mathscr{B}^{-}$, so $\mu\left(p_{0}+p_{1}^{1}\right)>\mu\left(p_{0}+q_{1}^{1}\right)$. A similar argument works for $p \geqslant q$. Hence, $\mu$ is compatible with $\geqslant$, as we wanted to show.

For the next theorem, and only for it, notice that the conditions on $\mathscr{B}$ and $\succcurlyeq$ have been changed.

THEOREM 5.8. Let $\mathscr{B}$ be a o-complete Boolean algebra and $\geqslant$ a binary relation with field $\mathscr{B}$ such that:

(i) Every finite subalgebra admits a probability measure compatible with $\geqslant$.

(ii) If for every $i, p_{i} \subseteq p_{i+1} \preccurlyeq q$, then $\cup_{i<\infty} p_{i} \preccurlyeq q$. Under these conditions there is $a$ probability measure $\mu$ such that

(a) If there is a $p \in \mathscr{B}$, such that for every $q \subseteq p$ there is a $q^{\prime} \subseteq q$ with $0 \prec q^{\prime} \prec q$, then $\mu$ is compatible with $\geqslant$.

(b) If for every $p \in \mathscr{B}$ there is a $q \subseteq p$ such that $q^{\prime} \subseteq q$ implies $q^{\prime} \sim q$, or $q^{\prime} \sim 0$, then $\mu$ is weakly compatible with $\geqslant$.

Proof. This theorem is easily reduced to the preceding one by considering the Boolean algebra $\mathscr{B}^{\prime}=\mathscr{B} / I$, where $\mathscr{B} / I$ is the quotient algebra obtained from $\mathscr{B}$ modulo the $\sigma$-ideal of elements equivalent to $0, I$.

6. Examples and special cases. We now turn our attention to some additional restrictions on $\Gamma$ which yield compatible measures, and an example by Steve Leth showing that some $\Gamma$ force compatible measures to assign irrational numbers to the atoms. 
EXAmple 6.1 (Steve LeTh). There is a $\Gamma$ having a unique compatible measure $\mu$ such that $\mu a_{n}$ is irrational for all $n$.

Let $\Gamma^{-}$contain the equalities $b_{n} \approx T_{n}$ for $n=0,1,2, \ldots$, where $b_{n}=a_{2 n}+a_{2 n+1}$ and $T_{n}=\sum_{n+1}^{\infty} b_{i}$. Clearly, if $\mu$ is compatible with $\Gamma^{-}$then $\mu b_{n}=1 / 2^{n+1}$. For each $n$ choose an irrational $c_{n} \in\left(1 / 2^{n+2}-1 / 2^{n+4}, 1 / 2^{n+2}\right)$ and let $d_{n}=1 / 2^{n+1}-c_{n}$. Clearly, $c_{0}>d_{0}>c_{1}>d_{1}>c_{2}>d_{2}>\cdots$. Each $c$ can be expressed as a sum $\sum\left\{2^{-i}: i \in I_{n}\right\}$, and each $d_{n}$ can be expressed as a sum $\Sigma\left\{2^{-j}: j \in J_{n}\right\}$. We now add to $\Gamma^{-}$all equalities of the form $a_{2 n} \approx \Sigma\left\{a_{i}: i \in I_{n}\right\}$ and $a_{2 n+1} \approx \Sigma\left\{a_{j}: j \in J_{n}\right\}$. Clearly, there is a unique measure $\mu$ compatible with $\Gamma^{-}$, and $\mu a_{2 n}=c_{n}$ and $\mu a_{2 n+1}=d_{n}$ for all $n$. Now $\Gamma^{-}$is completed to a total preordering $\Gamma$ in the obvious way.

THEOREM 6.2. If $a_{n} \preccurlyeq S_{n}$ for all $n$ then there is a probability measure compatible with $\succcurlyeq$.

Proof OF 6.2. We need several lemmas.

LEMMA 6.3. For every atom $a_{k}$ there is $a q \subseteq S_{k}$ such that $a_{k} \approx q$.

Proof. Let $a_{n_{0}}=a_{k+1}$ and, if $a_{k}>\Sigma^{s} a_{n_{i}}$, let $a_{n_{s+1}}$ be the atom of lowest index such that $a_{k}>\Sigma^{s+1} a_{n_{i}}$. Let $q=\sum^{\infty} a_{n_{i}}$. We claim that $q \approx a_{k}$. Surely $q \preccurlyeq a_{k}$ by monotonicity. If $q \prec a_{k}$ then $q \supseteq S_{n}$ for some $n$ by Lemma 3.1 and the definition of $q$. Let $n^{*}$ be the least $n$ such that $q \supseteq S_{n}$. Note $n^{*} \neq k$ since then $q \approx S_{k}$, but $a_{k} \preccurlyeq S_{k}$. But if $n^{*}>k$ then $a_{n^{*}} \not q$ and $a_{n^{*}} \preccurlyeq S_{n^{*}}$, contrary to the choice of the $a_{n}$. Hence, $q \prec a_{k}$ is impossible, and we have $q \approx a_{k}$.

LEMMA 6.4. For every $a_{k}$ there is $a q \subseteq S_{k}$ such that $a_{k} \approx q$ and $q \supseteq a_{l}+a_{m}$ for some $l$ and $m$.

Proof. There are only finitely many $i$ such that $a_{k} \approx a_{i}$ (as shown in §2). Now apply 6.3 to the atom $a_{n_{i}}$ of highest index.

LEMMA 6.5. For every $p$ there is some $q$ such that $p \approx q$ and $q \supseteq a_{n}$ for infinitely many $n$.

Proof. If $p=p^{1}+a_{k_{0}}$, where $p^{1} \subseteq a_{0}+a_{1}+\cdots+a_{k_{0}-1}$, we apply 6.4 to $a_{k_{0}}$ getting $p \approx p^{1}+q_{0}$. If $q_{0}=q_{0}^{1}+a_{k_{1}}$, where $q_{0}^{1} \subseteq a_{0}+a_{1}+\cdots+a_{k_{1}-1}$, we apply 6.4 to $a_{k_{1}}$, getting $p=p^{1}+q_{0}^{1}+q_{1}$. We continue the procedure getting $q_{1}, q_{2}, \ldots$ and $q_{1}^{1}, q_{2}^{1}, \ldots$ such that $p=p^{1}+q_{0}^{1}+q_{1}^{1}+\cdots+q_{n}$. If some $q_{n} \supseteq a_{l}$ for infinitely many $l$, we are done. If not, we let $q=p^{1}+q_{0}^{1}+q_{1}^{1}+\cdots$. We claim that $p \approx q$. Clearly,

$$
q \cup S_{n} \supseteq p^{1}+q_{0}^{1}+q_{1}^{1}+\cdots+q_{l} \approx p
$$

for all $l$ and $n$, so by Property II, $q \geqslant p$. On the other hand, for all $n$ there is an $l$ such that $q \cap \tilde{S_{n}} \subseteq p^{1}+q_{0}^{1}+q_{1}^{1}+\cdots+q_{l} \approx p$, so by Property II, $q \preccurlyeq p$. It follows that $q \approx p$ as needed.

For the existence of a weakly compatible measure $\mu$ we apply 5.3. In fact $\mu$ is a strongly compatible measure. For suppose $p>r$. By 5.3(iii) if $r \nsupseteq S_{n}$ for all $n$, then $\mu p>\mu r$. On the other hand, if $r \supseteq S_{n}$ for some $n$, we use 6.5 to get $q \approx p$ with 
$q \supseteq a_{k}$ for infinitely many $k$. There are $q_{1}, q^{*}, r_{1}$ such that $q=q_{1}+q^{*}$ and $r=r_{1}+q^{*}$. By 5.3(iii) $\mu q_{1}>\mu r_{1}$, from which it follows that $\mu q>\mu r$ and $\mu p>\mu r$. This shows that $\mu$ is compatible with $\Gamma$.

We suspect that the condition $a_{n} \leqslant S_{n}$ for all $n$ implies the uniqueness of the compatible measure, but we do not see how to prove or disprove this.

THEOREM 6.6. Suppose that $a_{n} \geqslant S_{n}$ for all $n$. Let $\sum r_{i}$ be a series of real numbers such that:

(i) $\sum r_{i}=1$;

(ii) $r_{n}=\sum_{i=n} r_{i+1}$ whenever $a_{n} \approx S_{n}$;

(iii) $r_{n}>\sum_{i=n} r_{i+1}$ whenever $a_{n}>S_{n}$.

Let $\mu p=\Sigma\left\{r_{i}: a_{i} \subseteq p\right\}$. Then $\mu$ is a probability measure compatible with $\geqslant$. In particular, if $a_{n} \geqslant S_{n}$ for all $n$ and $a_{k} \succ S_{k}$ for at least one $k$, then there are infinitely many probability measures compatible with $\succcurlyeq$.

Proof. Let $\mu$ be as above and suppose $p>q$. Without loss of generality we can suppose $p \subseteq \tilde{q}$. Let $m$ be the least $n$ such that $a_{n} \subseteq p$ or $a_{n} \subseteq q$. Since $a_{m} \geqslant S_{m}$ and either $p \subseteq S_{m}$ or $q \subseteq S_{m}$, we must have $a_{m} \subseteq p$. Then $\mu p \geqslant \mu a_{m} \geqslant \mu S_{m} \geqslant \mu q$. If $\mu p=\mu q$ then $q=S_{m}$ and $p=a_{m}$ (by the elementary properties of the series $\sum r_{i}$ ), so $a_{m} \approx S_{m}$ by (ii) and (iii)-contradiction. Hence $\mu p>\mu q$.

An easy modification of the argument just given shows that if $p \approx q$ then $\mu p=\mu q$.

At this time we are unable to prove that if $a_{n} \geqslant S_{n}$ for all $n$ greater than some $n^{*}$ then $\geqslant$ has a compatible probability measure.

7. Problems and remarks. We conclude with some open questions and a few remarks concerning our interest in them.

Are the conditions on $\Gamma$ (i.e., finite satisfiability and monotonicity) sufficient for the existence of a compatible measure? If not, then a counterexample might lead to additional conditions, algebraic or analytic in flavor, that are sufficient.

This paper considers only total (i.e., complete) preorderings, where every pair of elements $p, q \in \mathscr{B}$ are comparable, $p \preccurlyeq q$ or $q \preccurlyeq p$. There are several reasons why it is desirable to extend these results to partial preorderings where not every pair $p, q$ can be compared. Intuitively, we have no way of describing all of the uncountably many events (subsets of $\mathscr{B}$ ), never mind comparing them. Moreover, in many concrete situations our interest is only in a finite or countably infinite set of events, and a measure compatible with these events would be sufficient. Secondly, generalizations of this kind open the way to mathematical applications in which different measures have to be extended or combined.

A further step in generalizing the results from total preorderings to partial preorderings is to demand of some of the events that they be assigned particular numerical values. Intuitively, some of the events might be simple enough to determine a probability while others might be so complicated that only comparative probabilities can be given initially. Thus a compatible measure would assign to some of the events their predetermined value and would honor all the comparisons in $\Gamma$ as well. 
There are some cases, for example if $a_{n} \approx S_{n}$ for all $n$, when $\Gamma$ admits only one compatible measure. There are other cases, for example when $a_{n}>S_{n}$ for all $n$, when $\Gamma$ admits many compatible measures. Can we characterize those $\Gamma$ that admit a unique compatible measure, or at least can we find very general conditions which assure a unique compatible measure?

Let $\mathscr{B}^{-}$be the subalgebra of the atomic Boolean algebra $\mathscr{B}$ generated by the finite Boolean operations from the atoms of $\mathscr{B}, a_{0}, a_{1}, a_{2}, \ldots$ Let $\Gamma^{-}$be the set of equalities and inequalities of $\Gamma$ which mention only the elements of $\mathscr{B}^{-}$. If $\mu^{-}$is a measure on $\mathscr{B}^{-}$compatible with $\Gamma^{-}$then $\mu^{-}$is uniquely extendable to a probability measure $\mu$ on $\mathscr{B}$ honoring $\Gamma$ (an application of the Carathéodory Extension Theorem). Notice that $\Gamma^{-}$is countable, so we can talk about a recursive or recursively enumerable $\Gamma^{-}$. Several problems now arise naturally. If $\Gamma^{-}$is recursive or recursively enumerable and a compatible measure exists, is there a recursive compatible measure? (Here we want a recursive function $f(n, m)$ such that $f(n, m)$ is the $m$ th decimal place in $\mu a_{n}$, or a function $f(n, m)$ such that when $n=p_{i_{1}} p_{i_{2}} \cdots p_{i_{k}}$, where the $p$ 's are distinct primes, then $f(n, m)$ is the $m$ th decimal place in $a_{i_{1}}+a_{i_{2}}$ $+\cdots+a_{i_{k}}$ )

ADDED IN PROOF. Steve Leth has recently proved that the condition $a_{n} \leqslant S_{n}$ for all $n$ implies that the compatible measure of Theorem 6.2 is unique, answering the question posed after the proof of 6.2. He also constructed a counterexample to the third problem of $\$ 7$ in which one term is assigned a fixed value and no compatible measure exists.

\section{REFERENCES}

[KLST] D. H. Krantz, R. D. Luce, P. Suppes and A. Tversky, Foundations of measurement, Vol. I, Academic Press, New York, 1971.

[V] C. Villegas, On qualitative probability o-algebras, Ann. Math. Statist. 35 (1964), 1787-1796.

[SC] M. G. Schwarze and R. Chuaqui, $\sigma$-additive measurement structures, Mathematical Logic in Latin America (Arruda, Chuaqui and da Costa, eds.), North-Holland, Amsterdam, 1980, pp. 351-364.

[KPS] C. H. Kraft, J. W. Pratt and A. Seidenberg, Intuitive probability on finite sets, Ann. Math. Statist. 30 (1959), 408-419.

Department of Mathematics, Universidad Catolica de Chile, Casilla 114-D, Santiago, Chile

Department of Mathematics, University of Colorado, Boulder, Colorado 80309 